\title{
Testing generalized $C P$ symmetries with precision studies at DUNE
}

\author{
Newton Nath, ${ }^{1,2, *}$ Rahul Srivastava, ${ }^{3, \dagger}$ and José W. F. Valle ${ }^{3, *}$ \\ ${ }^{1}$ Institute of High Energy Physics, Chinese Academy of Sciences, Beijing, 100049, China \\ ${ }^{2}$ School of Physical Sciences, University of Chinese Academy of Sciences, Beijing, 100049, China \\ ${ }^{3}$ AHEP Group, Institut de Física Corpuscular-CSIC/Universitat de València, Parc Científic de Paterna, \\ C/Catedrático José Beltrán, 2 E-46980 Paterna (Valencia)—Spain
}

(Received 27 November 2018; published 8 April 2019)

\begin{abstract}
We examine the capabilities of the DUNE experiment in probing leptonic $C P$ violation within the framework of theories with generalized $C P$ symmetries characterized by the texture zeros of the corresponding $C P$ transformation matrices. We investigate DUNE's potential to probe the two least known oscillation parameters, the atmospheric mixing angle $\theta_{23}$ and the Dirac $C P$ phase $\delta_{C P}$. We fix theory-motivated benchmarks for $\left(\sin ^{2} \theta_{23}, \delta_{C P}\right)$ and take them as true values in our simulations. Assuming 3.5 years of neutrino running plus 3.5 years in the antineutrino mode, we show that in all cases DUNE can significantly constrain and in certain cases rule out the generalized $C P$ texture zero patterns.
\end{abstract}

DOI: 10.1103/PhysRevD.99.075005

\section{INTRODUCTION}

The discovery of oscillations $[1,2]$ provides a major milestone in the development of particle physics over the past few decades [3]. Several fundamental open issues in cosmology may also be closely related to the lepton sector and the properties of neutrinos, which we are just starting to uncover. For example, neutrinos could hold the key to the mystery associated with the origin of the baryon asymmetry of the Universe. Although this could arise within the Standard Model through anomalous electroweak baryonnumber nonconserving processes [4], the mechanism on its own turns out not to be realistic [5]. However, sphaleron processes can convert a preexisting lepton number, producing a net baryon number, a process called leptogenesis [6]. Indeed, this mechanism could, in principle, account for the observed matter to antimatter asymmetry of the Universe provided $C P$ is violated in the lepton sector in an adequate manner. This brings the issue of $C P$ violation in the neutrino sector to the spotlight. By exploring the phenomenon of neutrino oscillations, the Deep Underground Neutrino Experiment (DUNE) [7] aims to improve our understanding of neutrinos through the study of one of the three $C P$ phases present in the simplest theories of massive neutrinos [8].

\footnotetext{
*newton@ihep.ac.cn †rahulsri@ific.uv.es

*valle@ific.uv.es
}

Published by the American Physical Society under the terms of the Creative Commons Attribution 4.0 International license. Further distribution of this work must maintain attribution to the author(s) and the published article's title, journal citation, and DOI. Funded by SCOAP ${ }^{3}$.
DUNE is an international experiment for neutrino studies that will consist of two neutrino detectors placed in the world's most intense neutrino beam. The Long-Baseline Neutrino Facility will provide the neutrino beam line, while the two detectors will play complementary roles. These detectors will have the capability of searching for new $C P$ violating features of neutrino oscillations, thereby probing the existence of leptonic $C P$ violation. There have already been symmetry-based studies in the context of the DUNE experiment performed, e.g., in Refs. [9-13]. In particular, we note that popular flavor symmetry frameworks with $\mu-\tau$ symmetry, such as those in Refs. [14-17] (see Ref. [18] for a recent review and references) usually predict $\theta_{13}=0$ (zero reactor mixing), and also $\theta_{23}, \delta_{C P}$ values at odds with current global neutrino oscillation studies [19]. This led to revamped theories with viable predictions [20].

In this paper we reexamine the sensitivities of the DUNE experiment in probing leptonic $C P$ violation. Rather than considering specific full-fledged neutrino theories from first principles, here we adopt a model-independent framework based on generalized $C P$ symmetries. These are characterized by the pattern of texture zeros of the corresponding generalized $C P$ transformation matrices [21]. Apart from its own theoretical significance, a study based on generalized $C P$ symmetries is important and timely, as this class of theories can naturally yield consistent values of the oscillation parameters, while retaining predictive power [22-24]. The paper is structured as follows. In Secs. II and III we describe the generalized $C P$ matrices and their texture zeros, while in Sec. IV we briefly describe our numerical simulation. Results are presented in Sec. V and a brief summary with further discussion is given in Sec. VI. 


\section{GENERALIZED CP SYMMETRY}

In this section we provide a brief overview of the generalized $C P$ method and the phenomenological consequences of $C P$ texture zeros. We will mainly follow the notation of Refs. $[21,22,25]$. We start with the definition of the generalized $C P$ symmetries. The generalized $C P$ transformations $X_{\psi}$ acting on a particular fermionic field $\psi$ are defined as follows:

$$
\psi \stackrel{C P}{\mapsto} i X_{\psi} \gamma^{0} \mathcal{C} \bar{\psi}^{T} .
$$

The associated $C P$ transformation matrix $X_{\psi}$ of Eq. (1) can be deemed to be a valid symmetry of the mass term provided it satisfies

$X_{\psi}^{T} m_{\psi} X_{\psi}=m_{\psi}^{*}, \quad$ for Majorana fields,

$X_{\psi}^{\dagger} M_{\psi}^{2} X_{\psi}=M_{\psi}^{2 *}, \quad$ for Dirac fields, where $M_{\psi}^{2} \equiv m_{\psi}^{\dagger} m_{\psi}$.

The mass matrix $m_{\psi}$ of a Majorana fermion can be diagonalized by a unitary transformation $U_{\psi}$; e.g., for a $3 \times 3$ Majorana mass matrix we have

$U_{\psi}^{T} m_{\psi} U_{\psi}=\operatorname{diag}\left(m_{1}, m_{2}, m_{3}\right)$, for Majorana fields,

while for Dirac fermions one needs a biunitary transformation to diagonalize the mass matrix. However, the "squared mass matrix" $M_{\psi}^{2}$ of a Dirac fermion can also be diagonalized by a unitary transformation. For the $3 \times 3$ Dirac case we get

$U_{\psi}^{\dagger} M_{\psi}^{2} U_{\psi}=\operatorname{diag}\left(m_{1}^{2}, m_{2}^{2}, m_{3}^{2}\right), \quad$ for Dirac fields.

Note that while writing down Eqs. (4) and (5) we have assumed nondegenerate masses, i.e., $m_{1} \neq m_{2} \neq m_{3}$. From Eqs. (2)-(5), one can show that the unitary matrix $U_{\psi}$ diagonalizing a mass matrix invariant under a given generalized $C P$ symmetry $X_{\psi}$, satisfies the following constraint:

$U_{\psi}^{\dagger} X_{\psi} U_{\psi}^{*} \equiv P= \begin{cases}\operatorname{diag}( \pm 1, \pm 1, \pm 1), & \text { for Majorana fields } \\ \operatorname{diag}\left(e^{i \delta_{1}}, e^{i \delta_{2}}, e^{i \delta_{3}}\right), & \text { for Dirac fields }\end{cases}$

where $e^{i \delta_{i}} ; i=1,2,3$ are independent phases. ${ }^{1}$ It is also easy to show that $X_{\psi}$ is always a symmetric matrix [25]. Owing to its symmetric nature, it can be "Takagi decomposed" as

\footnotetext{
${ }^{1}$ Notice that even for Majorana fermions if some of the masses are zero then the corresponding " \pm " entry should be replaced by a complex phase.
}

$$
X_{\psi}=\Sigma \cdot \Sigma^{T}
$$

From Eqs. (6) and (7) we find that

$$
P^{-\frac{1}{2}} U_{\psi}^{\dagger} \Sigma \equiv O_{3},
$$

where $O_{3}$ is a real $3 \times 3$ orthogonal matrix. Equation (8) implies that the unitary matrix $U_{\psi}$ can be written in terms of the $C P$ matrix $\Sigma$ as

$$
U_{\psi}=\Sigma O_{3}^{T} P^{-\frac{1}{2}}
$$

The orthogonal matrix $O_{3}$ can be parametrized in terms of three rotation angles as follows:

$$
\begin{aligned}
O_{3}= & \left(\begin{array}{ccc}
1 & 0 & 0 \\
0 & \cos \theta_{1} & \sin \theta_{1} \\
0 & -\sin \theta_{1} & \cos \theta_{1}
\end{array}\right)\left(\begin{array}{ccc}
\cos \theta_{2} & 0 & \sin \theta_{2} \\
0 & 1 & 0 \\
-\sin \theta_{2} & 0 & \cos \theta_{2}
\end{array}\right) \\
& \times\left(\begin{array}{ccc}
\cos \theta_{3} & \sin \theta_{3} & 0 \\
-\sin \theta_{3} & \cos \theta_{3} & 0 \\
0 & 0 & 1
\end{array}\right)
\end{aligned}
$$

where the three angles $\theta_{i} ; i=1,2,3$ are free parameters whose values can be constrained by experiments. In the rest of this work we will impose generalized $C P$ symmetries on the neutrino mass matrix in order to constrain leptonic mixing parameters. For definiteness we work in the charged lepton diagonal basis.

Before moving forward we should remark on some salient features of the generalized $C P$ symmetry approach. The first thing to note is that the fermion masses are not constrained by the generalized $C P$ symmetries and in almost all cases free parameters can be chosen to match the observed masses. The real predictive power of generalized $C P$ symmetries lies in their ability to predict the fermion mixing angles and phases.

Another point to understand is the difference between the cases when neutrinos are Dirac or Majorana in nature. In this work we will mainly assume neutrinos to be Majorana particles, though that need not be the case [26-30]. If neutrinos are Dirac particles then it can be shown that [24]:

(i) The mixing parameters testable in neutrino oscillations experiments, i.e., solar $\left(\theta_{12}\right)$, reactor $\left(\theta_{13}\right)$, and atmospheric $\left(\theta_{23}\right)$ angles, as well as the $C P$ phase $\left(\delta_{C P}\right)$ will be identical both for Majorana or Dirac neutrinos.

(ii) For Majorana neutrinos, the generalized $C P$ symmetries in general also impose nontrivial constraints on the "Majorana phases." In the Dirac neutrino case, the Majorana phases are unphysical and can be rotated away by appropriate field redefinitions [8]. 
It follows that all of our discussion in this work regarding the potential of the DUNE experiment to probe $C P$ texture zeros holds equally well for the case of Dirac neutrinos. This is to be expected as it is well known that oscillation experiments are insensitive to the Dirac or Majorana nature of neutrinos [31].

\section{TEXTURE ZEROS OF GENERALIZED $C P$ MATRICES}

In this section we look at the phenomenological consequences of all the possible texture zeros allowed in the generalized $C P$ matrix $X$. As we will discuss, the presence of texture zeros in $X$ has implications for both oscillation parameters as well as for the Majorana phases, leading to implications also for neutrinoless double beta decay experiments. Since in this work we are mainly interested in the potential of DUNE to probe $C P$ texture zeros, we will only focus on the implications on parameters relevant to neutrino oscillations, namely, the three angles and the Dirac $C P$ phase $\delta_{C P}$.

The $X$ matrices can be classified by the number of the textures zeros present in a given $X$. Notice that any $C P$ matrix $X$ with more than four texture zeros is not phenomenologically viable. The various possible texture zeros matrices with four or lesser texture zeros are listed in Table I. Below we briefly summarize their predictions for oscillation parameters. For further details and other implications of $C P$ texture zeros see Ref. [21] whose results we now summarize.

\section{A. Four texture zeros}

As mentioned before, the maximum number phenomenologically viable texture zeros $X$ can have is four. There are in total three possible four texture zero $C P$ matrices as shown in Table I. However, among them only one $X_{I}$ is consistent with current neutrino oscillation data. This $C P$ matrix is nothing but the $C P$ matrix corresponding to the famous $\mu-\tau$ symmetry $[16,17]$. It predicts

$$
\begin{array}{ll}
\sin ^{2} \theta_{13}=\sin ^{2} \theta_{2}, & \sin ^{2} \theta_{23}=\frac{1}{2}, \\
\sin ^{2} \theta_{12}=\sin ^{2} \theta_{3}, & \sin \delta_{C P}=\operatorname{sgn}\left(\sin \theta_{2} \sin 2 \theta_{3}\right),
\end{array}
$$

where the parameters $\theta_{i} ; i=1,2,3$ are the angles of the orthogonal matrix $\mathrm{O}_{3}$ in the parametrization of Eq. (10). Notice that $X_{I}$ predicts maximal value of the atmospheric angle as well as maximal value of the Dirac phase; i.e., $\sin ^{2} \theta_{23}=0.5$ and $\delta_{C P}= \pm \pi / 2$.

\section{B. Three texture zeros}

There is only one possibility for three texture zeros in the $X$ matrix, as shown in Table I. For the oscillation parameters it predicts

$$
\begin{array}{ll}
\sin ^{2} \theta_{13}=\sin ^{2} \theta_{2}, & \sin ^{2} \theta_{12}=\sin ^{2} \theta_{3}, \\
\sin ^{2} \theta_{23}=\sin ^{2} \theta_{1}, & \sin \delta_{C P}=0,
\end{array}
$$

where, as before, $\theta_{i} ; i=1,2,3$ are the angles of the orthogonal matrix $O_{3}$ in Eq. (10). Thus the three texture zero matrix $X_{I V}$ predict no $C P$ violation. Moreover, all the mixing angles in this case remain unconstrained. Allowed parameter space of $\sin ^{2} \theta_{23}-\delta_{C P}$ for this type are shown in Fig. 1 by the green line. In plotting Fig. 1 we have varied the free parameters $\theta_{i} ; i=1,2,3$ in their full allowed range of $(0,2 \pi]$, to obtain the mixing parameters through the relations given in Eq. (12). The $X_{I V}$ predict range shown in Fig. 1 is then obtained by requiring that all the mixing parameters should lie within their current $3 \sigma$ ranges [19]. The same procedure is followed throughout this section to obtain the correlation plots for $\sin ^{2} \theta_{23}$ and $\delta_{C P}$.

\section{Two texture zeros}

For two texture zeros there are in total three possibilities, as listed in Table I, all of which are phenomenologically viable. The first case of two texture zeros $X_{V}$ is nothing but generalized $\mu-\tau$ symmetry discussed in Ref. [22]. ${ }^{2}$ For oscillation parameters it predicts:

$$
\begin{aligned}
\sin ^{2} \theta_{13} & =\sin ^{2} \theta_{2}, \quad \sin ^{2} \theta_{12}=\sin ^{2} \theta_{3}, \\
\sin ^{2} \theta_{23} & =\frac{1}{2}\left(1-\cos \Theta \cos 2 \theta_{1}\right), \\
\sin \delta_{C P} & =\frac{\operatorname{sgn}\left(\sin \theta_{2} \sin 2 \theta_{3}\right) \sin \Theta}{\sqrt{1-\cos ^{2} \Theta \cos ^{2} 2 \theta_{1}}} .
\end{aligned}
$$

These expressions lead to a correlation,

$$
\sin ^{2} \delta_{C P} \sin ^{2} 2 \theta_{23}=\sin ^{2} \Theta,
$$

where $\Theta$ is a label that defines the $C P$ scenario. Within a given model the value of $\Theta$ is fixed. For example, taking $\Theta=\pi / 2$ in $X_{V}$ leads to exact $\mu-\tau$ reflection symmetry corresponding to the $X_{I}$ case. Taking $\Theta=0$ leads to a $C P$ conserving value of the Dirac $C P$ phase ${ }^{3}$ and allows $3 \sigma$ range of $\theta_{23}$. The $\sin ^{2} \theta_{23}-\delta_{C P}$ predictions for $\Theta=2 \pi / 17$ are shown in Fig. 2. For the sake of consistency and for ease of comparison, throughout this section we will take the representative value of $\Theta=2 \pi / 17$ for showing the $\sin ^{2} \theta_{23}-\delta_{C P}$ predictions.

\footnotetext{
${ }^{2}$ Generalized $\mu-\tau$ symmetry in the charged lepton sector is discussed in Ref. [24]. Recently, a similar pattern using residual discrete symmetries has also been considered in Ref. [32].

${ }^{3}$ Note that $\delta_{C P}=0$ is now disfavored by current data at $3 \sigma$ whereas $\delta_{C P}=\pi$ is allowed at $2 \sigma$ [19].
} 
TABLE I. Possible $C P$ transformation matrices with their corresponding $\boldsymbol{\Sigma}$ matrices. Here $\Theta, \alpha, \beta$, and $\gamma$ are real free parameters characterizing these $C P$ transformations. We adopt short-hand notations $c_{\Theta} \equiv \cos \Theta, s_{\Theta} \equiv \sin \Theta, \quad \alpha_{1} \equiv-\alpha+\beta+\gamma$, and $\alpha_{2} \equiv-\alpha+\beta+\gamma / 2$. Note that not all $C P$ texture zeros are phenomenologically viable (see text for details).

Possible $C P$ texture zeros

Four texture zeros

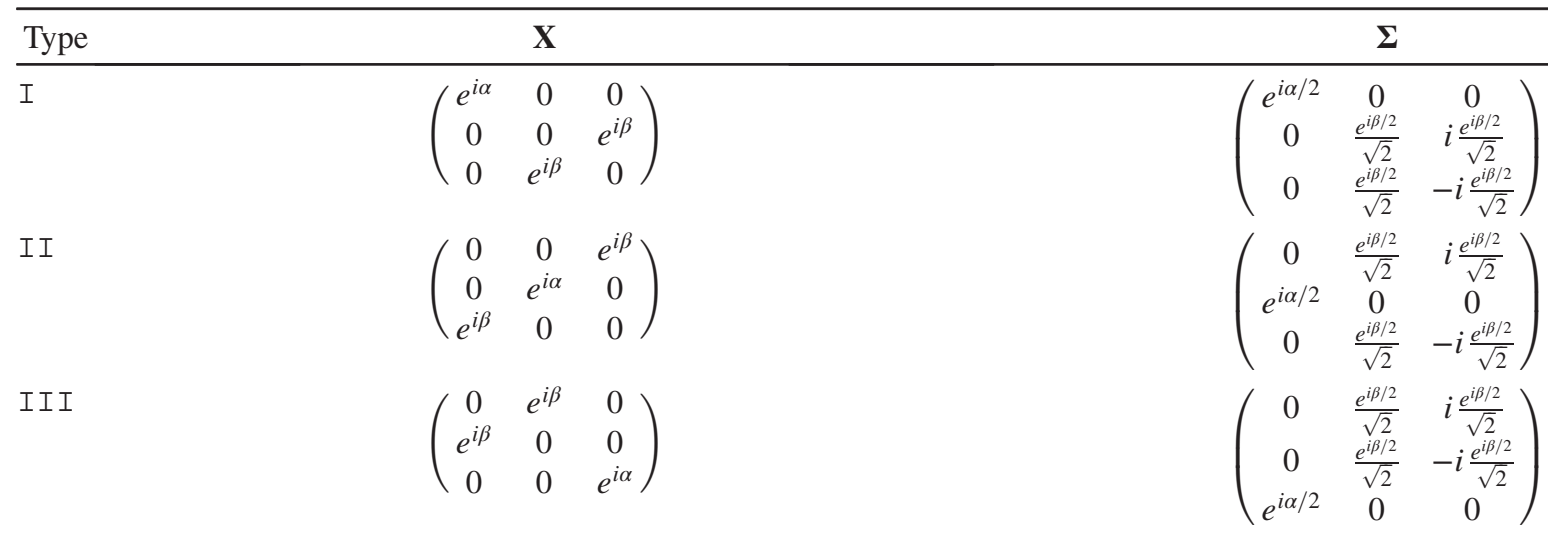

Three texture zeros IV

$$
\left(\begin{array}{ccc}
e^{i \alpha} & 0 & 0 \\
0 & e^{i \beta} & 0 \\
0 & 0 & e^{i \gamma}
\end{array}\right)
$$$$
\left(\begin{array}{ccc}
e^{i \alpha / 2} & 0 & 0 \\
0 & e^{i \beta / 2} & 0 \\
0 & 0 & e^{i \gamma / 2}
\end{array}\right)
$$

Two texture zeros

$\mathrm{V}$

$$
\left(\begin{array}{ccc}
e^{i \alpha} & 0 & 0 \\
0 & e^{i \beta} c_{\Theta} & i e^{i(\beta+\gamma) / 2} s_{\Theta} \\
0 & i e^{i(\beta+\gamma) / 2} s_{\Theta} & e^{i \gamma} c_{\Theta}
\end{array}\right)
$$

VI

$$
\left(\begin{array}{ccc}
e^{i \alpha} c_{\Theta} & 0 & i e^{i(\alpha+\gamma) / 2} s_{\Theta} \\
0 & e^{i \beta} & 0 \\
i e^{(\alpha+\gamma) / 2} s_{\Theta} & 0 & e^{i \gamma} c_{\Theta}
\end{array}\right)
$$

VII

$$
\left(\begin{array}{ccc}
e^{i \alpha} c_{\Theta} & i e^{i(\alpha+\beta) / 2} s_{\Theta} & 0 \\
i e^{i(\alpha+\beta) / 2} s_{\Theta} & e^{i \beta} c_{\Theta} & 0 \\
0 & 0 & e^{i \gamma}
\end{array}\right)
$$

One texture zero

VIII

$$
\left(\begin{array}{ccc}
0 & e^{i \alpha} c_{\Theta} & e^{i \beta} s_{\Theta} \\
e^{i \alpha} c_{\Theta} & e^{i \gamma} s_{\Theta}^{2} & -e^{i \alpha_{1}} c_{\Theta} s_{\Theta} \\
e^{i \beta} s_{\Theta} & -e^{i \alpha_{1}} c_{\Theta} s_{\Theta} & e^{2 i \alpha_{2}} c_{\Theta}^{2}
\end{array}\right)
$$

IX

$$
\left(\begin{array}{ccc}
e^{i \alpha} c_{\Theta}^{2} & e^{i \beta} s_{\Theta} & e^{i \gamma} c_{\Theta} s_{\Theta} \\
e^{i \beta} s_{\Theta} & 0 & -e^{i \alpha_{1}} c_{\Theta} \\
e^{i \gamma} c_{\Theta} s_{\Theta} & -e^{i \alpha_{1}} c_{\Theta} & e^{i(-\alpha+2 \gamma)} s_{\Theta}^{2}
\end{array}\right)
$$

X

$$
\left(\begin{array}{ccc}
e^{i \alpha} c_{\Theta}^{2} & e^{i \gamma} c_{\Theta} s_{\Theta} & e^{i \beta} s_{\Theta} \\
e^{i \gamma} c_{\Theta} s_{\Theta} & e^{i(-\alpha+2 \gamma)} s_{\Theta}^{2} & -e^{i \alpha_{1}} c_{\Theta} \\
e^{i \beta} s_{\Theta} & -e^{i \alpha_{1}} c_{\Theta} & 0
\end{array}\right)
$$

Democratic $C P$ matrix

$\mathrm{XI}$

$$
\frac{1}{\sqrt{3}}\left(\begin{array}{ccc}
e^{i \alpha} & e^{i\left(\frac{\alpha+\beta}{2}+\frac{2 \pi}{3}\right)} & e^{i\left(\frac{\alpha+\gamma}{2}+\frac{2 \pi}{3}\right)} \\
e^{i\left(\frac{\alpha+\beta}{2}+\frac{2 \pi}{3}\right)} & e^{i \beta} & e^{i\left(\frac{\beta+\gamma}{2}+\frac{2 \pi}{3}\right)} \\
e^{i\left(\frac{\alpha+\gamma}{2}+\frac{2 \pi}{3}\right)} & e^{i\left(\frac{\beta+\gamma}{2}+\frac{2 \pi}{3}\right)} & e^{i \gamma}
\end{array}\right)
$$

$$
\begin{aligned}
& \left(\begin{array}{ccc}
e^{\frac{i \alpha}{2}} & 0 & 0 \\
0 & e^{\frac{i \beta}{2}} c_{\frac{\Theta}{2}} & i e^{\frac{i \beta}{2}} S_{\frac{\Theta}{2}} \\
0 & i e^{\frac{i \gamma}{2}} S_{\Theta} & e^{\frac{i \gamma}{2}} c_{\Theta}
\end{array}\right) \\
& \left(\begin{array}{ccc}
0 & e^{\frac{i \alpha}{2}} c_{\frac{\Theta}{2}} & i e^{\frac{i \alpha}{2}} S_{\frac{\Theta}{2}} \\
e^{\frac{i \beta}{2}} & 0 & 0 \\
0 & i e^{\frac{i \gamma}{2}} S_{\frac{\Theta}{2}} & e^{\frac{i \gamma}{2}} c_{\frac{\Theta}{2}}
\end{array}\right) \\
& \left(\begin{array}{ccc}
0 & e^{\frac{i \alpha}{2}} c_{\frac{\Theta}{2}} & i e^{\frac{i \alpha}{2}} S_{\frac{\Theta}{2}} \\
0 & i e^{\frac{i \beta}{2}} S_{\frac{\Theta}{2}} & e^{\frac{i \beta}{2}} c_{\frac{\Theta}{2}} \\
e^{\frac{i \gamma}{2}} & 0 & 0
\end{array}\right)
\end{aligned}
$$

$$
\begin{gathered}
\frac{1}{\sqrt{2}}\left(\begin{array}{ccc}
-i e^{i(\alpha-\gamma / 2)} & e^{i(\alpha-\gamma / 2)} & 0 \\
i e^{i \gamma / 2} c_{\Theta} & e^{i \gamma / 2} c_{\Theta} & \sqrt{2} e^{i \gamma / 2} s_{\Theta} \\
i e^{i \alpha_{2}} s_{\Theta} & e^{i \alpha_{2}} s_{\Theta} & -\sqrt{2} e^{i \alpha_{2}} c_{\Theta}
\end{array}\right) \\
\frac{1}{\sqrt{2}}\left(\begin{array}{ccc}
-i e^{i \alpha / 2} s_{\Theta} & -e^{i \alpha / 2} s_{\Theta} & \sqrt{2} e^{i \alpha / 2} c_{\Theta} \\
i e^{i(\beta-\alpha / 2)} & -e^{i(\beta-\alpha / 2)} & 0 \\
i e^{i(\gamma-\alpha / 2)} c_{\Theta} & e^{i(\gamma-\alpha / 2)} c_{\Theta} & \sqrt{2} e^{i(\gamma-\alpha / 2)} s_{\Theta}
\end{array}\right) \\
\frac{1}{\sqrt{2}}\left(\begin{array}{ccc}
-i e^{i \alpha / 2} s_{\Theta} & -e^{i \alpha / 2} s_{\Theta} & \sqrt{2} e^{i \alpha / 2} c_{\Theta} \\
i e^{i(\gamma-\alpha / 2)} c_{\Theta} & e^{i(\gamma-\alpha / 2)} c_{\Theta} & \sqrt{2} e^{i(\gamma-\alpha / 2)} s_{\Theta} \\
i e^{i(\beta-\alpha / 2)} & -e^{i(\beta-\alpha / 2)} & 0
\end{array}\right)
\end{gathered}
$$

$\operatorname{diag}\left(e^{i \alpha / 2}, e^{i \beta / 2}, e^{i \gamma / 2}\right) e^{-\frac{i \pi}{12}}\left(\begin{array}{ccc}\sqrt{\frac{2}{3}} & \frac{1}{\sqrt{3}} & 0 \\ \frac{-1}{\sqrt{6}} & \frac{1}{\sqrt{3}} & \frac{1}{\sqrt{2}} \\ \frac{-1}{\sqrt{6}} & \frac{1}{\sqrt{3}} & \frac{-1}{\sqrt{2}}\end{array}\right) \operatorname{diag}\left(1, e^{i \pi / 3}, 1\right)$ 


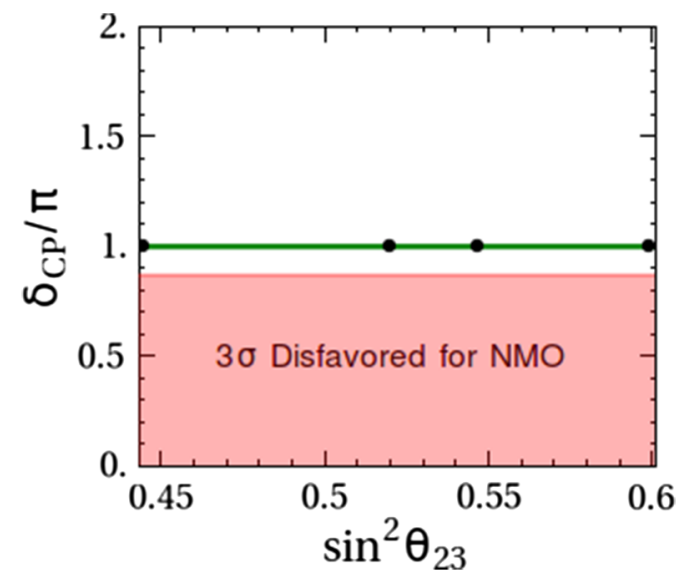

FIG. 1. The green line corresponds to the $\sin ^{2} \theta_{23}-\delta_{C P}$ prediction for $X_{I V}$ given in Eq. (12). The black points correspond to the benchmark values of Table II that lie within $X_{I V}$. The (red) shaded region is currently disfavored at $3 \sigma$ for normal mass ordering (NMO) [19].

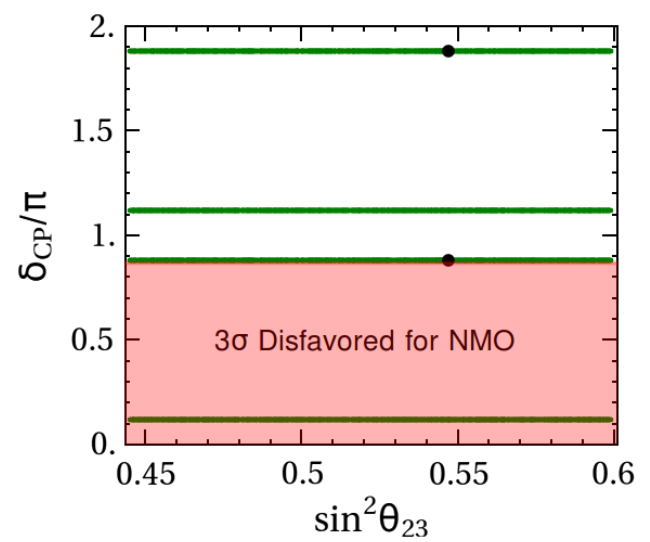

FIG. 2. The green lines correspond to the $\sin ^{2} \theta_{23}-\delta_{C P}$ prediction for $X_{V}$ given in Eq. (13). The black points correspond to the benchmark values of Table II that lie within $X_{V}$. The (red) shaded region is currently disfavored at $3 \sigma$ for normal mass ordering [19].

The second case of two texture zeros $X_{V I}$ predicts

$$
\begin{aligned}
\sin ^{2} \theta_{13} & =\frac{1}{2}\left(1-\cos \Theta \cos 2 \theta_{1}\right) \cos ^{2} \theta_{2}, \sin ^{2} \theta_{23}=\frac{2 \sin ^{2} \theta_{2}}{2-\left(1-\cos \Theta \cos 2 \theta_{1}\right) \cos ^{2} \theta_{2}}, \\
\sin ^{2} \theta_{12} & =\frac{\left(1+\cos \Theta \cos 2 \theta_{1}\right) \cos ^{2} \theta_{3}+\sin \theta_{2}\left[\left(1-\cos \Theta \cos 2 \theta_{1}\right) \sin \theta_{2} \sin ^{2} \theta_{3}-\cos \Theta \sin 2 \theta_{1} \sin 2 \theta_{3}\right]}{2-\left(1-\cos \Theta \cos 2 \theta_{1}\right) \cos ^{2} \theta_{2}}, \\
J_{C P} & =-\frac{1}{4} \sin \Theta \sin \theta_{2} \cos ^{2} \theta_{2} \sin 2 \theta_{3},
\end{aligned}
$$

where $J_{C P}=\frac{1}{8} \sin 2 \theta_{12} \sin 2 \theta_{23} \sin 2 \theta_{13} \cos \theta_{13} \sin \delta_{C P}$ is the standard $C P$ invariant.

The third possibility of two texture zeros $X_{V I I}$ is actually related with the $X_{V I}$ case by the exchange of the second and third rows. Thus the oscillation parameters in this case are same as for the $X_{V I}$ case given in Eq. (15), except that the angle $\theta_{23}$ and the Dirac phase $\delta_{C P}$ of Eq. (15) should be replaced by $\pi / 2-\theta_{23}$ and $\pi+\delta_{C P}$, respectively. In Fig. 3 we show the allowed parameter space of $\sin ^{2} \theta_{23}-\delta_{C P}$ for $X_{V I}$ (left panel) and $X_{V I I}$ (right panel) for $\Theta=2 \pi / 17$.

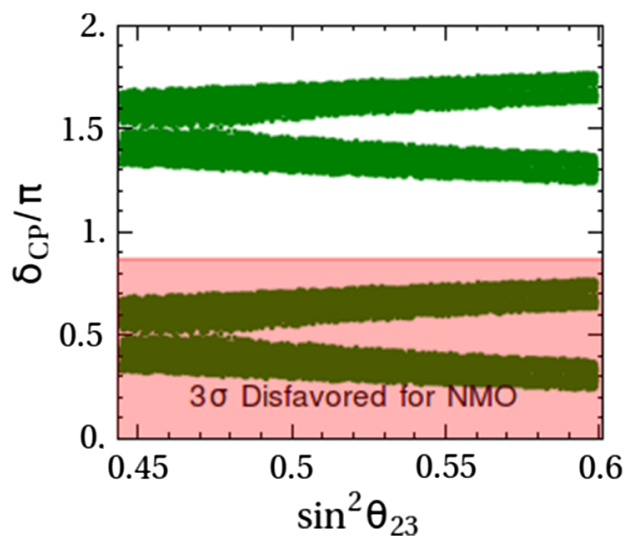

\section{One texture zero}

For the case of one texture zero there are again three distinct possibilities, as shown in Table I. However, out of these three, the $X_{V I I I} C P$ matrix cannot successfully account for current neutrino oscillation date. Hence, only two possibilities, i.e., $X_{I X}$ and $X_{X}$ are phenomenologically viable. The $C P$ matrix $X_{I X}$ predicts

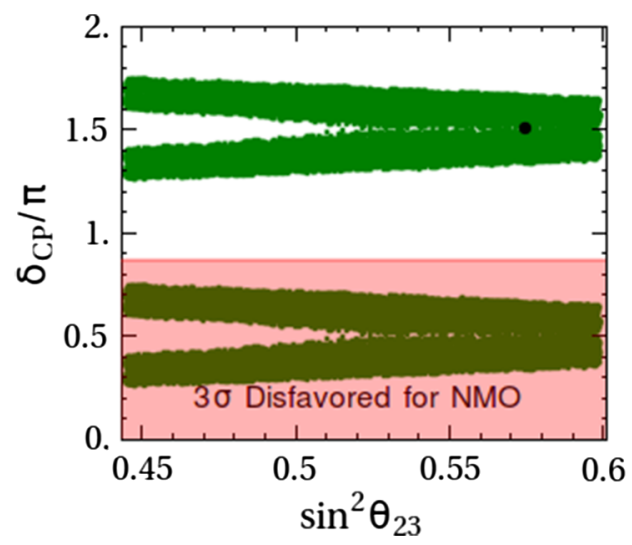

FIG. 3. Same as Fig. 2 but for $X_{V I}$ (left panel) and $X_{V I I}$ (right panel). 


$$
\begin{aligned}
\sin ^{2} \theta_{13}= & \frac{1}{4}\left[1+\cos ^{2} \theta_{1} \cos ^{2} \theta_{2}+\left(3 \cos ^{2} \theta_{1} \cos ^{2} \theta_{2}-1\right) \cos 2 \Theta-\sqrt{2} \sin 2 \theta_{1} \cos ^{2} \theta_{2} \sin 2 \Theta\right], \\
\sin ^{2} \theta_{12}= & 2\left[\left(\sqrt{2} \cos \Theta\left(\sin \theta_{1} \cos \theta_{3}+\cos \theta_{1} \sin \theta_{2} \sin \theta_{3}\right)+\sin \Theta\left(\cos \theta_{1} \cos \theta_{3}-\sin \theta_{1} \sin \theta_{2} \sin \theta_{3}\right)\right)^{2}\right. \\
& \left.+\cos ^{2} \theta_{2} \sin ^{2} \theta_{3} \sin ^{2} \Theta\right] /\left[3-\cos ^{2} \theta_{1} \cos ^{2} \theta_{2}+\left(1-3 \cos ^{2} \theta_{1} \cos ^{2} \theta_{2}\right) \cos 2 \Theta+\sqrt{2} \sin 2 \theta_{1} \cos ^{2} \theta_{2} \sin 2 \Theta\right], \\
\sin ^{2} \theta_{23}= & \frac{2\left(\sin ^{2} \theta_{2}+\sin ^{2} \theta_{1} \cos ^{2} \theta_{2}\right)}{3-\cos ^{2} \theta_{1} \cos ^{2} \theta_{2}+\left(1-3 \cos ^{2} \theta_{1} \cos ^{2} \theta_{2}\right) \cos 2 \Theta+\sqrt{2} \sin 2 \theta_{1} \cos ^{2} \theta_{2} \sin 2 \Theta}, \\
J_{C P}= & \frac{1}{16} \cos \theta_{1} \cos \theta_{2}\left[4 \sin 2 \theta_{1} \sin \theta_{2} \cos 2 \theta_{3}-\sin 2 \theta_{3}\left(1-3 \cos 2 \theta_{1}+2 \cos ^{2} \theta_{1} \cos 2 \theta_{2}\right)\right] \cos 2 \Theta \\
& +\frac{1}{128 \sqrt{2}}\left[\left(12 \cos ^{2} \theta_{1} \sin \theta_{1} \cos 3 \theta_{2}+\left(\sin \theta_{1}-15 \sin 3 \theta_{1}\right) \cos \theta_{2}\right) \sin 2 \theta_{3}\right. \\
& \left.+4\left(\cos \theta_{1}+3 \cos 3 \theta_{1}\right) \sin 2 \theta_{2} \cos 2 \theta_{3}\right] \sin 2 \Theta .
\end{aligned}
$$

Moreover, the $C P$ matrix $X_{X}$ is related to $X_{I X}$ by the exchange of the second and third rows. As a consequence, its oscillation parameters are the same as those in Eq. (16), except that $\sin ^{2} \theta_{23} \rightarrow 1-\sin ^{2} \theta_{23}$ and $\delta_{C P} \rightarrow \pi+\delta_{C P}$. The prediction for $\sin ^{2} \theta_{23}$ and $\delta_{C P}$ for the $C P$ matrices $X_{X}$ and $X_{I X}$ are shown in Fig. 4, respectively, taking $\Theta=2 \pi / 17$.

\section{E. Democratic $C P$ matrix}

Finally, one can also have $C P$ matrices without any texture zeros at all. There are infinite such possible matrices. A simple and predictive example of one such matrix is the so-called democratic $C P$ matrix, as shown in Table I. There are in total four such possible democratic $C P$ matrices but it can be shown that they all lead to the same mixing parameters, up to unphysical phase redefinitions [21]. Thus, in Table I we have only shown one such democratic matrix, namely, $X_{X I}$. It predicts

$$
\begin{aligned}
\sin ^{2} \theta_{13}= & \frac{1}{6}\left[4 \sin ^{2} \theta_{2}+\sqrt{2} \sin 2 \theta_{2} \sin \theta_{1}+2 \sin ^{2} \theta_{1} \cos ^{2} \theta_{2}\right], \\
\sin ^{2} \theta_{12}= & \left\{\left[\sin \theta_{1}\left(\sqrt{2} \sin 2 \theta_{2}-2 \sin \theta_{1} \sin ^{2} \theta_{2}\right)-4 \cos ^{2} \theta_{2}\right] \sin ^{2} \theta_{3}-2 \cos ^{2} \theta_{1} \cos ^{2} \theta_{3}+\left[\sin 2 \theta_{1} \sin \theta_{2}\right.\right. \\
& \left.\left.-\sqrt{2} \cos \theta_{1} \cos \theta_{2}\right] \sin 2 \theta_{3}\right\} /\left(4 \sin ^{2} \theta_{2}+\sqrt{2} \sin 2 \theta_{2} \sin \theta_{1}+2 \sin ^{2} \theta_{1} \cos ^{2} \theta_{2}-6\right), \\
\sin ^{2} \theta_{23}= & \frac{\sin 2 \theta_{2}\left(\sqrt{2} \sin \theta_{1}+2 \sqrt{3} \cos \theta_{1}\right)-2 \sin ^{2} \theta_{2}-\cos ^{2} \theta_{2}\left(\sqrt{6} \sin 2 \theta_{1}+\cos 2 \theta_{1}+5\right)}{2\left(4 \sin ^{2} \theta_{2}+\sqrt{2} \sin 2 \theta_{2} \sin \theta_{1}+2 \sin ^{2} \theta_{1} \cos ^{2} \theta_{2}-6\right)} \\
J_{C P}= & \frac{-1}{48 \sqrt{2}}\left\{\left[4 \sqrt{2} \sin 2 \theta_{2} \sin ^{2} \theta_{1} \cos \theta_{1}+4 \sin 2 \theta_{1} \cos 2 \theta_{2}\right] \cos 2 \theta_{3}+\left[5 \sin \theta_{2} \sin ^{2} \theta_{1}\right.\right. \\
& \left.\left.+\sqrt{2} \sin \theta_{1}\left(5 \cos ^{2} \theta_{1}-1\right) \cos \theta_{2}+\left(3 \cos ^{2} \theta_{1}+1\right) \sin 3 \theta_{2}+\sqrt{2} \sin ^{3} \theta_{1} \cos 3 \theta_{2}\right] \sin 2 \theta_{3}\right\} .
\end{aligned}
$$
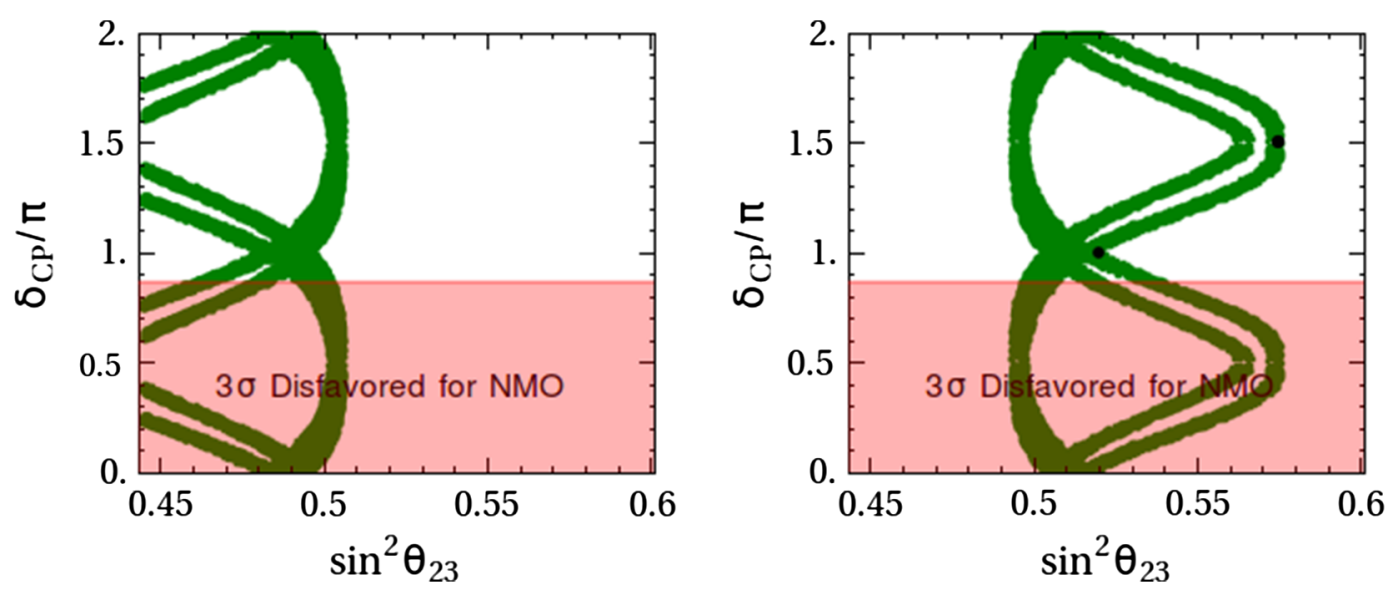

FIG. 4. Same as Fig. 2 but for $X_{I X}$ (left panel) and $X_{X}$ (right panel). 


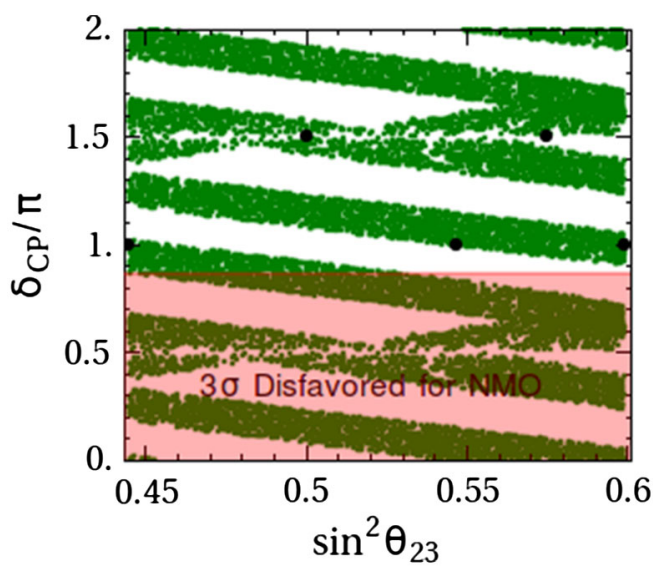

FIG. 5. Same as Fig. 2 but for $X_{X I}$.

In Fig. 5, we show the prediction for $\sin ^{2} \theta_{23}$ and $\delta_{C P}$ corresponding to the $C P$ matrix $X_{X I}$ with $\Theta=2 \pi / 17$.

In the following sections we will look at the potential of the upcoming Deep Underground Neutrino Experiment (DUNE) to probe the predictions of the above texture zero $C P$ matrices, focusing on the atmospheric angle $\theta_{23}$ and the Dirac $C P$ phase $\delta_{C P}$.

\section{EXPERIMENTAL AND SIMULATION DETAILS}

DUNE is a proposed next generation superbeam neutrino oscillation experiment at Fermilab, USA [33,34]. The DUNE collaboration has planned to utilize existing neutrinos from the main injector (NuMI) beam line design at Fermilab as a neutrino source. The far detector of DUNE will be installed at Sanford Underground Research Facility (SURF) in Lead, South Dakota. The distance from the source to the far detector is about $1300 \mathrm{~km}(810 \mathrm{mi})$ and it will be kept about $1.5 \mathrm{~km}$ under the surface. The first detector will record particle interactions near the source of the beam, at Fermilab, while the second, much larger, underground detector will use four $10 \mathrm{kton}$ volume of liquid argon time-projection chambers (LArTPC). The expected design flux corresponds to $1.07 \mathrm{MW}$ beam power which gives $1.47 \times 10^{21}$ protons on target $(\mathrm{POT})$ per year for an $80 \mathrm{GeV}$ proton beam energy.

For the numerical simulation of the DUNE data, we use the GLOBES package [35,36] along with the required auxiliary files presented in Ref. [34]. We perform our numerical analysis considering 3.5 years running time in both neutrino and antineutrino modes. Throughout this work, we consider 40 kton detector volume. While performing the numerical simulation, we have also taken into account both the appearance and disappearance channels of neutrinos as well as antineutrinos. In addition, we adopt signal and background normalization uncertainties for the appearance and disappearance channels as mentioned in the DUNE CDR [34].
Given that the latest global-fit values of neutrino oscillation parameters prefer normal neutrino mass ordering (i.e., $m_{1}<m_{2}<m_{3}$ ) over inverted neutrino mass ordering (i.e., $m_{3}<m_{1} \sim m_{2}$ ) at more than $3 \sigma$ [19,37], we focus our study on the first scenario. We adopt given "theory motivated" benchmark values for $\theta_{23}, \delta_{C P}$ as true values, and determine the resulting DUNE sensitivity regions. Concerning the former, we take the values suggested by our generalized $C P$ theories, as characterized by the corresponding $C P$ texture zeros. We fix solar oscillation parameters $\theta_{12}, \Delta m_{21}^{2}$ at their current best-fit values in both true and test. On the other hand, we assume current best-fit values for $\theta_{13}$ and $\Delta m_{31}^{2}$ as their true values, whereas in the test, we marginalize over their $3 \sigma$ ranges allowed by the latest global-fit results of Ref. [19]. In the next section, we provide a detailed description of our numerical analysis for different true benchmark points in the context of DUNE.

Before moving on to analyze the potential of the DUNE experiment to probe various specific $C P$ texture-zero patterns, we briefly quantify DUNE's general capability in probing leptonic $C P$ violation. To do that we assume, for definiteness, that the current indications from neutrino oscillation data survive, i.e., we fix as a benchmark the current value obtained in Ref. [19]. In Fig. 6 we compare our current determination of the leptonic $C P$ violating phase $\delta_{C P}$ with the expected range of $\delta_{C P}$ by the end of the $3.5+3.5$ run period of the DUNE experiment.

From Fig. 6 we see that if the current best-fit $C P$ value $\delta_{C P}=1.32 \pi$ is indeed the true value, then after $3.5+$ $3.5 \mathrm{yr}$ of running the DUNE experiment there will be a significant improvement in the measurement of $\delta_{C P}$. Currently at $3 \sigma$ the $\delta_{C P}$ lies in the range $(0.87-1.94) \pi$ [19], as indicated by the blue curve. However, at end of the $3.5+3.5 \mathrm{yr}$ of DUNE run the $3 \sigma$ will shrink appreciably down to $(1.08-1.68) \pi$, as seen in the red curve. Clearly

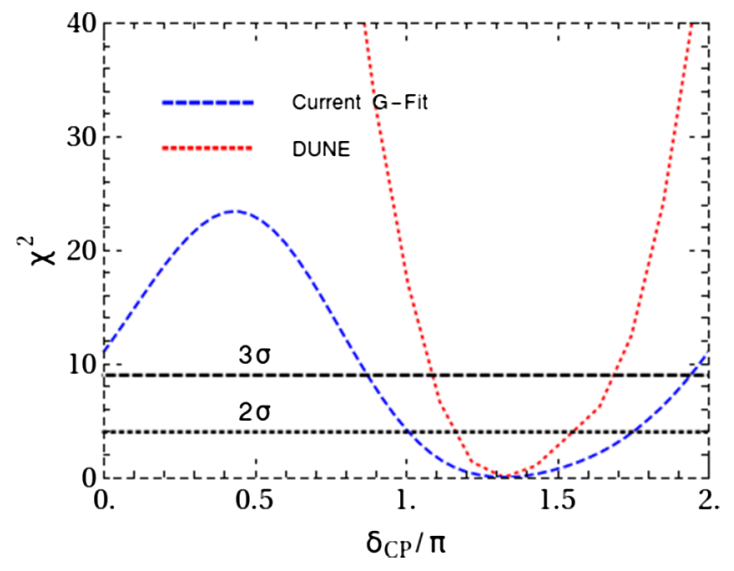

FIG. 6. Sensitivity of DUNE vs current global fit to probe leptonic $C P$ violation. In order to compare the two we have taken the current best-fit value, $\left(\sin ^{2} \theta_{23}, \delta_{C P}\right)=(0.547,1.32 \pi)$ from Ref. [19] as the true value for our simulations. The DUNE sensitivity is given after $3.5+3.5 \mathrm{yr}$ of runtime. 
the DUNE experiment offers good prospects for testing leptonic $C P$ violation. Similar improvement is also expected in the measurement of the atmospheric angle $\sin ^{2} \theta_{23}$. In Sec. $\mathrm{V}$ we investigate the capabilities of the DUNE experiment to test various theory predictions for $\sin ^{2} \theta_{23}$ and $\delta_{C P}$ obtained within the framework of generalized $C P$ symmetry theories.

\section{CONSEQUENCES OF GENERALIZED CP SYMMETRY AT DUNE}

Having discussed the theory predictions originating from texture zeros in the generalized $C P$ matrices discussed in Sec. III, we now investigate DUNE's capability to test these predictions. We note that in most cases texture zeros yield regions in the $\theta_{23}-\delta_{C P}$ plane, rather than a unique point. ${ }^{4}$ Although the nature of the predicted $\theta_{23}-\delta_{C P}$ correlations differ in each case, they often lead to overlapping regions in the $\theta_{23}-\delta_{C P}$ plane. Hence, in this section we exploit this fact by adequately choosing benchmarks that are shared by several $C P$ texture zero patterns. By employing this strategy we aim to cover all viable cases with as few representative benchmark points as possible, as listed in Table II. These benchmark values are carefully chosen in such a way that

(i) They are well motivated from the theoretical point of view and are indeed realized in at least one of the cases discussed in Sec. III.

(ii) They can be used as simulated true values against which other $C P$ texture zero cases (those not including it) can be tested.

(iii) They are such that all the $C P$ texture zero cases of Sec. III can be covered with as few benchmark points as possible.

Table II summarizes our benchmark points. The second column represents the benchmark values we have adopted, while the third column indicates the generalized $C P$ texture zero patterns in which they appear. Notice also that, while theory motivated, all of the benchmark points in Table II are also taken to lie within $3 \sigma$ of the currently allowed values [19]. The corresponding results of our DUNE simulations are shown in Figs. 7-9.

We now proceed to discuss the phenomenological implications of the different benchmark values as given by Table II. In Figs. 7 and 8, we describe the predicted $\left(\sin ^{2} \theta_{23}, \delta_{C P}\right)$ sensitivity regions for different benchmark points, for the case of normal neutrino mass ordering. Different color variations show $\chi^{2}$ values ranging from $\chi^{2}=0$ all the way up to $\chi^{2}=40$ (see figure label for details). We have also drawn the contours for $2 \sigma$ (blackdotted) and $3 \sigma$ (black-solid), corresponding to $\chi^{2}=6.18$, 11.83 for 2 d.o.f, respectively. The "black-dot" points

\footnotetext{
${ }^{4}$ An exception is our texture zero $C P$ matrix $X_{I}$, which corresponds to $\mu-\tau$ symmetry and predicts a unique point.
}

TABLE II. Set of benchmark values corresponding to all the possible $C P$ textures zeros. Note that the case BP-I represents exact $\mu-\tau$ reflection symmetry. Here, BP stands for "benchmark point," whereas BL implies "benchmark line."

\begin{tabular}{lcc}
\hline \hline Scenarios & $\left(\sin ^{2} \theta_{23}, \delta_{C P}\right)$ & $C P$ textures \\
\hline Benchmark points & $(0.5,1.5 \pi)$ & $X_{I}, X_{V}, X_{V I}, X_{X I}$ \\
BP-I & $(0.547, \pi)$ & $X_{I V}, X_{V}, X_{X I}$ \\
BP-II & $(0.445, \pi)$ & $X_{I V}, X_{V}, X_{X I}$ \\
BP-III & $(0.599, \pi)$ & $X_{I V}, X_{V}, X_{X I}$ \\
BP-IV & $(0.52, \pi)$ & $X_{I V}, X_{V}, X_{X}$ \\
BP-V & $(0.575,1.5 \pi)$ & $X_{X}, X_{X I}$ \\
BP-VI & $(0.547,0.88 \pi)$ & $X_{V}$ \\
BP-VII & $(0.547,1.88 \pi)$ & $X_{V}$ \\
BP-VIII & $(0.445 \oplus 0.599, \pi)$ & $X_{I V}, X_{V}, X_{X}, X_{X I}$ \\
Benchmark lines & $X_{I}, X_{V}, X_{V I}, X_{X}, X_{X I}$ \\
BL-I & $(0.445 \oplus 0.599,1.5 \pi)$ & \\
BL-II & & \\
\hline \hline
\end{tabular}

depict the true benchmark values for different scenarios, as given by Table II. For quick comparison with our current knowledge of these parameters, in all figures, we have also shown as a red star the current best-fit value, $\left(\sin ^{2} \theta_{23}, \delta_{C P}\right)=(0.547,1.32 \pi)$ from Ref. [19].

Figure 7(a) shows the DUNE sensitivity regions for the BP-I scenario. Recall that this scenario corresponds to having exact $\mu-\tau$ reflection symmetry, and predicts the parameters $\theta_{23}$ and $\delta_{C P}$ both to be maximal. In plotting Fig. 7(a) we have taken these values as true benchmark points. One sees that after $3.5 \mathrm{yr}$ running time in both neutrino and antineutrino modes, DUNE will probe a large part of the $\theta_{23}, \delta_{C P}$ plane at the $3 \sigma$ level. As shown in Table II, this benchmark value is also shared by the scenarios associated with the $C P$ matrices of $X_{V}, X_{V I}$, $X_{X I}$. As clear from Fig. 7(a), for this BP-I case, apart from a small region, DUNE will have the capability to significantly rule out the $C P$ conservation hypothesis, i.e., $\delta_{C P}=0, \pi$.

The DUNE simulation performed by taking the second benchmark BP-II as true value is shown in Fig. 7(b). This benchmark point lies in the allowed ranges for the $X_{I V}, X_{V}$, $X_{X I} C P$ matrices, as shown in Table II. Since this benchmark value corresponds to $C P$ conservation, it can test for the maximal $C P$ violation hypothesis. As shown in Fig. 7(b), in this case maximal $C P$ violation can indeed be excluded at more than $3 \sigma$. Furthermore, the allowed range for the atmospheric angle can also be significantly constrained.

Our determinations of DUNE's reach for benchmarks BP-III and BP-IV are shown in Figs. 7(c) and 7(d), respectively. As stated in Table II, these benchmarks are also shared with $X_{I V}, X_{V}, X_{X I} C P$ zero texture patterns. While both correspond to $C P$ conserving scenarios, these benchmarks have significantly different values of the atmospheric angle; BP-III corresponding to $\sin ^{2} \theta_{23}$ in 


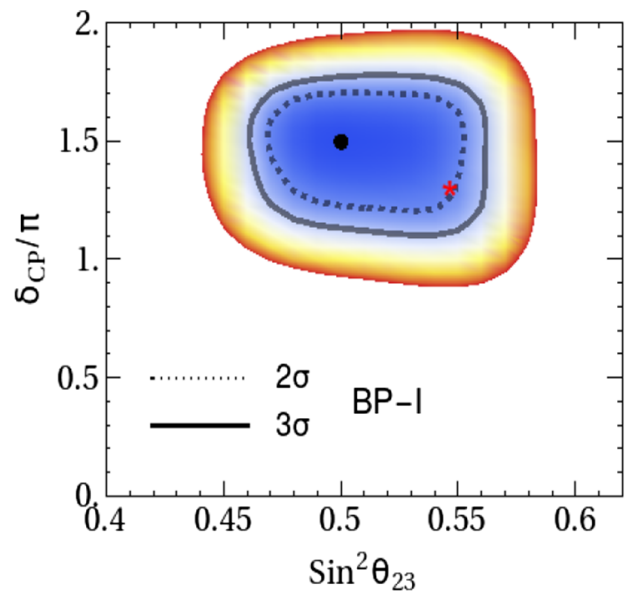

(a) BP-I: $\sin ^{2} \theta_{23}=0.5, \delta_{\mathrm{CP}}=1.5 \pi$.

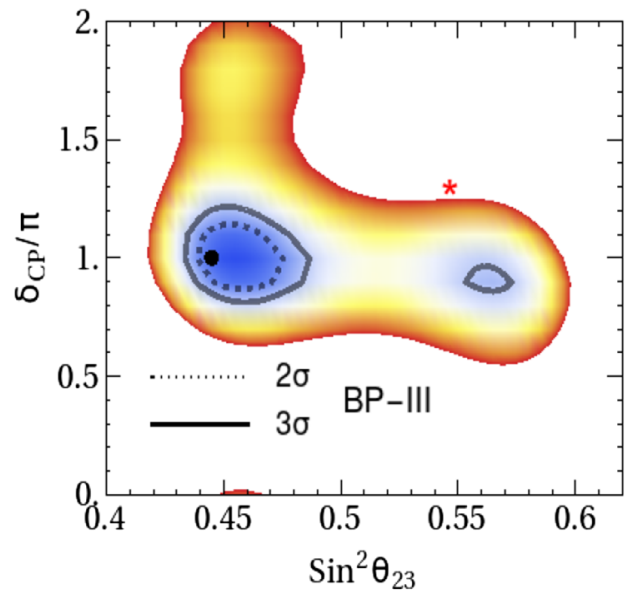

(c) BP-III: $\sin ^{2} \theta_{23}=0.445, \delta_{\mathrm{CP}}=\pi$.

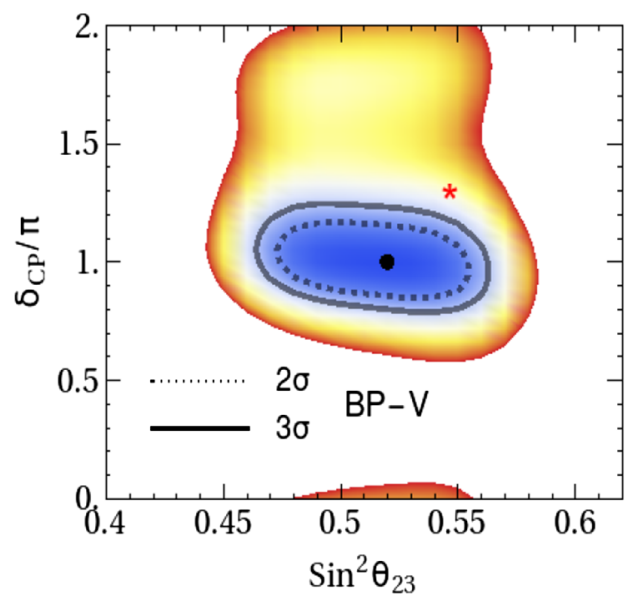

(e) BP-V: $\sin ^{2} \theta_{23}=0.52, \delta_{\mathrm{CP}}=\pi$.

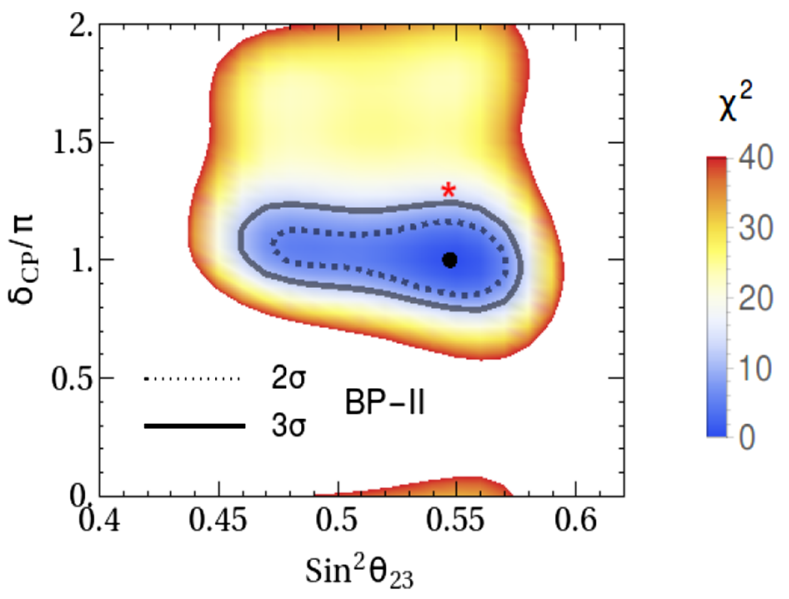

(b) BP-II: $\sin ^{2} \theta_{23}=0.547, \delta_{\mathrm{CP}}=\pi$.

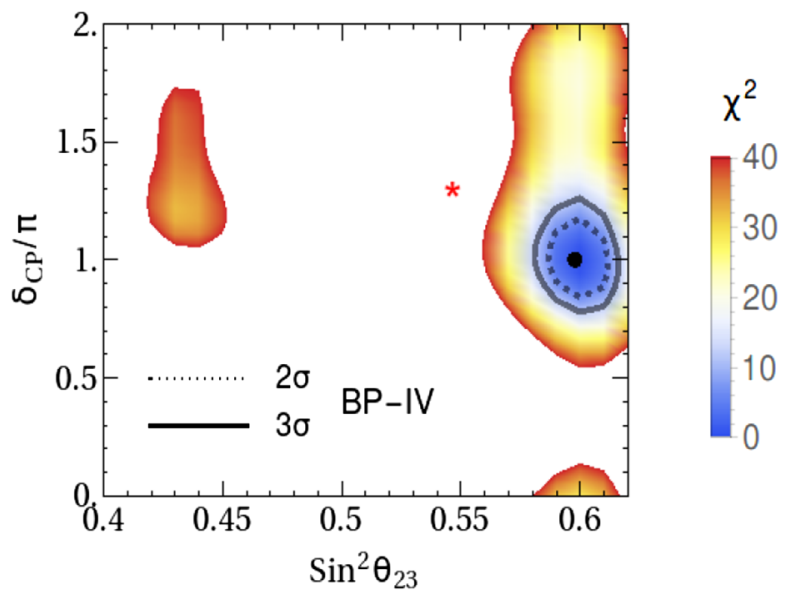

(d) BP-IV: $\sin ^{2} \theta_{23}=0.599, \delta_{\mathrm{CP}}=\pi$.

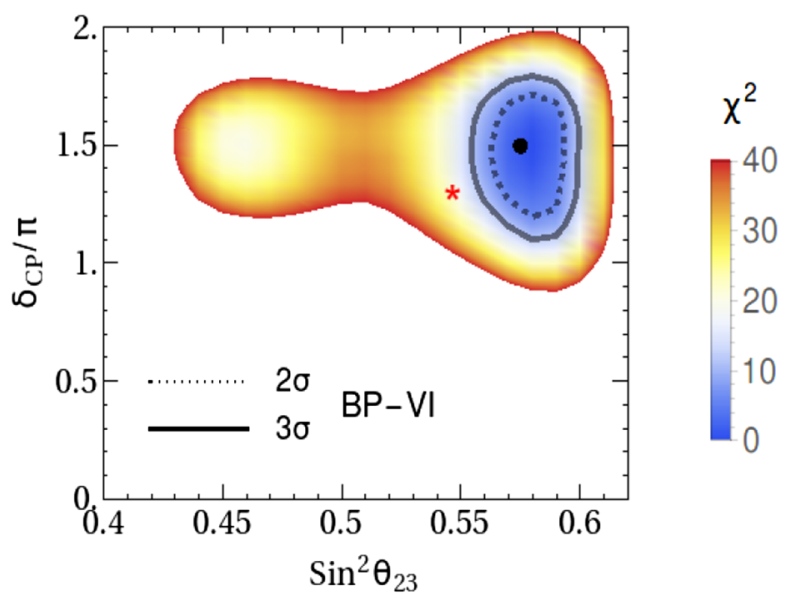

(f) BP-VI: $\sin ^{2} \theta_{23}=0.575, \delta_{\mathrm{CP}}=1.5 \pi$.

FIG. 7. Sensitivity regions for DUNE in the $\left(\sin ^{2} \theta_{23}, \delta_{C P}\right)$ plane for different true benchmark points (i.e., BP-I, BP-II, BP-III, BP-IV, BP-V, and BP-VI) as given in Table II. The black dots represent true benchmark values of $\left(\sin ^{2} \theta_{23}, \delta_{C P}\right)$, whereas the red star is the current best-fit value, $\left(\sin ^{2} \theta_{23}, \delta_{C P}\right)=(0.547,1.32 \pi)$ from Ref. [19]. 


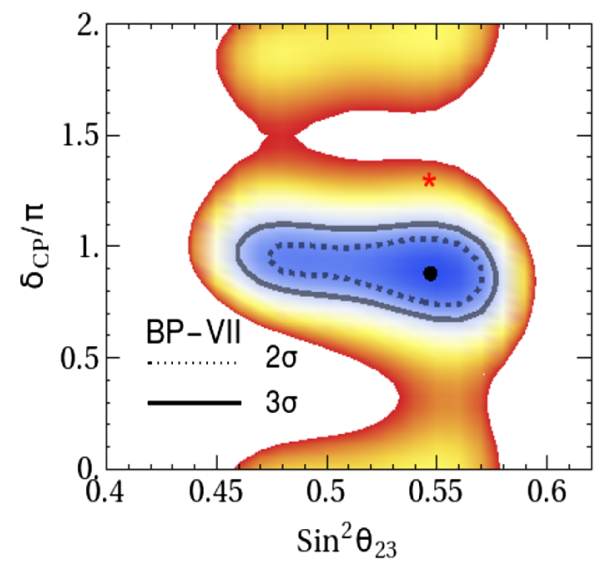

(a) BP-VII: $\sin ^{2} \theta_{23}=0.547, \delta_{\mathrm{CP}}=0.88 \pi$.

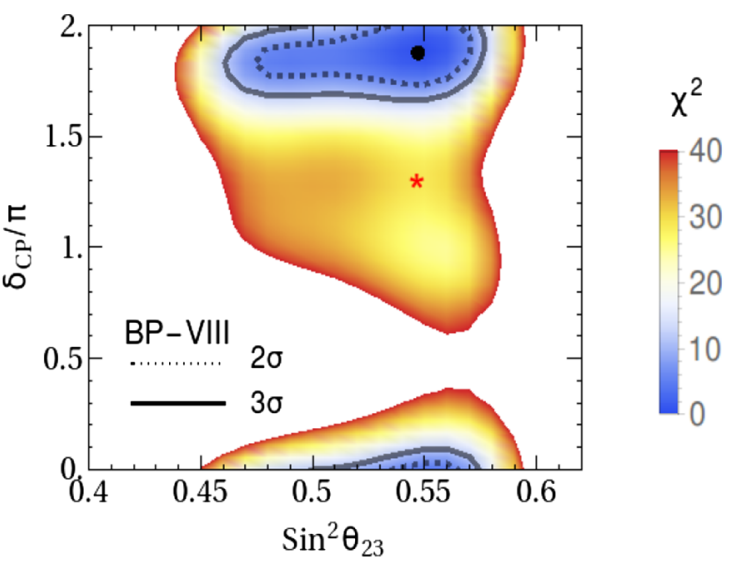

(b) BP-VIII: $\sin ^{2} \theta_{23}=0.547, \delta_{\mathrm{CP}}=1.88 \pi$.

FIG. 8. DUNE sensitivity regions in the $\left(\sin ^{2} \theta_{23}, \delta_{C P}\right)$ plane for benchmark points BP-VII and BP-VIII, as given in Table II. The black dots (red stars) represent true benchmark (global best-fit) values of $\left(\sin ^{2} \theta_{23}, \delta_{C P}\right)$.

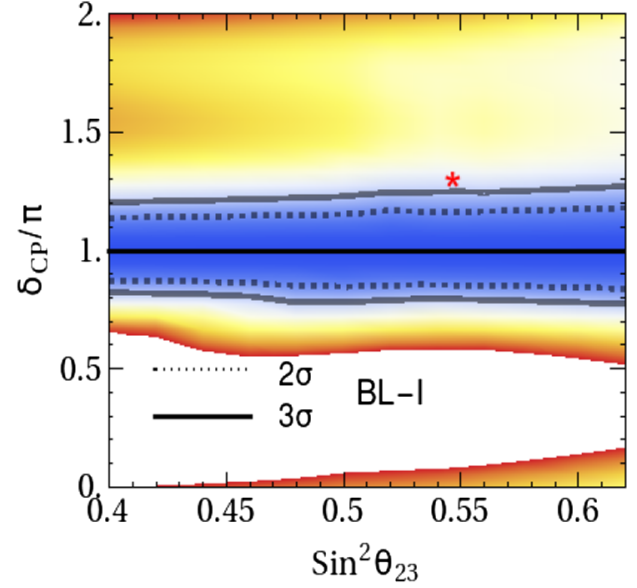

(a) BL-I: $\sin ^{2} \theta_{23}=0.445 \oplus 0.599, \delta_{\mathrm{CP}}=\pi$.

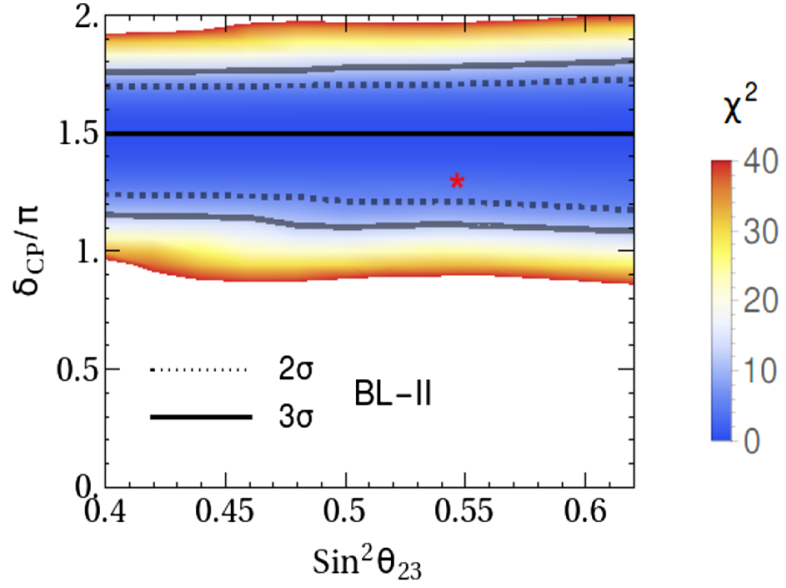

(b) BL-II: $\sin ^{2} \theta_{23}=0.445 \oplus 0.599, \delta_{\mathrm{CP}}=1.5 \pi$.

FIG. 9. Allowed parameter space of DUNE in $\left(\sin ^{2} \theta_{23}, \delta_{C P}\right)$ plane for the different true benchmark line as given in Table II. Also, the red star represents global best-fit value of $\left(\sin ^{2} \theta_{23}, \delta_{C P}\right)$. Here, left (right) panel corresponds to $C P$ conserving (maximal $C P$ violating) value of true $\delta_{C P}$.

the lower octant $\left(\sin ^{2} \theta_{23}<0.5\right)$, while BP-IV has $\sin ^{2} \theta_{23}$ in the upper octant $\left(\sin ^{2} \theta_{23}>0.5\right)$. Just like BP-II, these benchmarks are again $C P$ conserving, and will probe the hypothesis of maximal $C P$ violation, which will be ruled out at more than $3 \sigma$. One also sees from these panels that DUNE will restrict the allowed $\sin ^{2} \theta_{23}$ range to a small region around its true value. As shown in Fig. 7(c), for BP-III DUNE will exclude maximal atmospheric mixing to a very high significance, while the whole higher octant, will be disfavored at $2 \sigma$. A small region around $\sin ^{2} \theta_{23} \sim$ 0.56 will remain allowed at $3 \sigma$. For BP-IV one sees, from Fig. 7(d), that DUNE will rule out maximal atmospheric angle at $\chi^{2} \sim 40$. Moreover, DUNE will also exclude the whole lower octant, $\theta_{23}$ at more than $3 \sigma$. For such benchmark values, the exact $\mu-\tau$ reflection symmetry, i.e., $\left(\sin ^{2} \theta_{23}, \delta_{C P}\right)=(0.5,1.5 \pi)$ will be excluded by DUNE at $\chi^{2} \sim 40$.

The results of our DUNE simulations for benchmarks BP-V and BP-VI are shown in Figs. 7(e) and 7(f), respectively. As shown in Table II these benchmark points are shared by the $C P$ matrices $X_{X}, X_{X I}$. To quantify DUNE's sensitivities in this case, in Figs. 7(e) and 7(f) we adopt $C P$ conserving and maximal $C P$ violating true benchmark values, respectively. Note that benchmark BP-V is $C P$ conserving, with $\sin ^{2} \theta_{23}=0.52$. Maximal $C P$ violation can be ruled out to a very high significance in this case, whereas the maximal atmospheric mixing is allowed within $2 \sigma$; see Fig. $7(\mathrm{e})$. In contrast, benchmark 

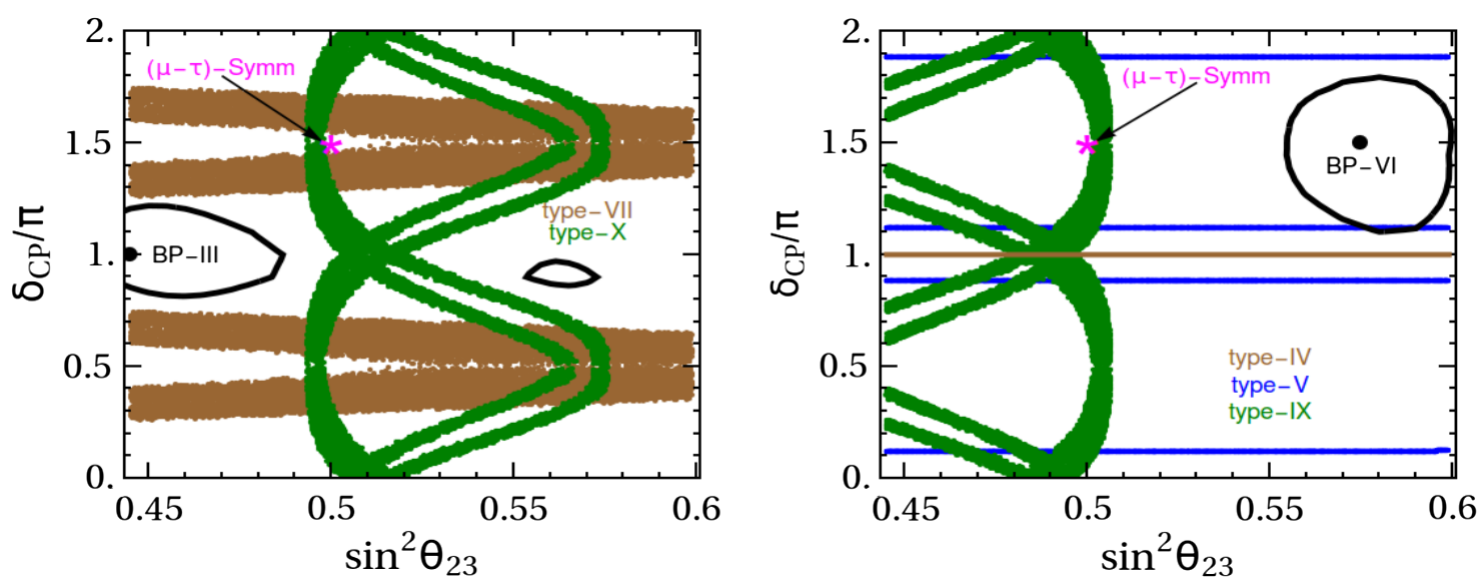

FIG. 10. Allowed parameter space in $\sin ^{2} \theta_{23}-\delta_{C P}$ plane for DUNE at $3 \sigma$ for BP-III (left) and BP-VI (right) panel as shown by black contours, respectively. Also, scatter points show correlation between $\sin ^{2} \theta_{23}$ and $\delta_{C P}$ for different $C P$ matrices.

BP-VI has maximal $C P$ violation $\left(\delta_{C P}=1.5 \pi\right)$ and nonmaximal atmospheric mixing $\left(\sin ^{2} \theta_{23}=0.575\right)$. As seen from Fig. 7(f) in this case DUNE will have the potential to exclude the $C P$ conservation hypothesis at $\chi^{2} \sim 40$. We also notice from BP-VI [see Fig. 7(f)] that DUNE will be able to rule out the lower octant of the atmospheric angle with more than $3 \sigma$ sensitivity. Moreover, one sees that in this case the determination of $\theta_{23}, \delta_{C P}$ improves considerably.

We now turn to the case of benchmarks BP-VII and BPVIII, which appear associated with the $X_{V}, X_{X I} C P$ matrix patterns. Notice that so far all the benchmark points we have considered (see Table II and Fig. 7) were either $C P$ conserving or violated $C P$ maximally. The current benchmark points $\mathrm{BP}-\mathrm{VII}$ and $\mathrm{BP}-\mathrm{VIII}$ violate $C P$ nonmaximally. BP-VII has nonmaximal $C P$ violation and nonmaximal atmospheric mixing, with $\delta_{C P}=0.88 \pi$ and $\sin ^{2} \theta_{23}=0.547$. The results of our DUNE simulation for this benchmark are shown in Fig. 8(a). In this case maximal $C P$ violation will be excluded well above $3 \sigma$. In contrast, $C P$ conservation cannot be excluded even at $2 \sigma$. Likewise, maximal atmospheric mixing also cannot be ruled out. The results of our DUNE simulation for the final benchmark point, BP-VIII, corresponding to $\sin ^{2} \theta_{23}=0.547$ and $\delta_{C P}=1.88 \pi$, are shown in Fig. 8(b). Here too we find that maximal $C P$ violation can be ruled out at more than $3 \sigma$. However, no $C P$ violation as well as maximal atmospheric mixing remain allowed within $2 \sigma$. Notice that for BP-VII [see Fig. 8(a)] the exact $\mu-\tau$ reflection symmetry can be ruled out at $\chi^{2} \sim 40$, while for BP-VIII it can only be ruled out at the $3 \sigma$ level, as seen from Fig. 8(b). Notice also that, except for Fig. 7(a) which corresponds to the BP-I case, the current best-fit value shown in the plots by the "red star" lies outside the $3 \sigma$ range in all cases of Figs. 7 and 8.

Having discussed at length the results of our DUNE simulations for the theory-motivated benchmark points in Table II, we now discuss two examples where the benchmarks are given by lines, ${ }^{5}$ as seen in the last two rows in Table II. The results are shown in Fig. 9. To perform the numerical analysis for Fig. 9, we have taken several true-value points all along the "true-value line." The truevalue points are taken close enough to each other so that they effectively form a true-value line. Then, taking one true-value point at a time, we perform our DUNE simulations for the test values for that point. This is done by marginalizing over the test values of $\theta_{23}$ in its allowed $3 \sigma$ range and calculating minimum values of $\chi^{2}$ (i.e., $\chi_{\min }^{2}$ ). Finally, we collect all the $\chi_{\min }^{2}$ for each true value of $\theta_{23}$ and show the results in the $\left(\sin ^{2} \theta_{23}, \delta_{C P}\right)$ plane. In Fig. 9(a) we have taken the $C P$ conserving true benchmark value $\delta_{C P}=\pi$, whereas in Fig. 9(b) we focused on the benchmark value $\delta_{C P}=1.5 \pi$ with maximal $C P$ violation, as true value. In both cases, $3 \sigma$ ranges of $\theta_{23}$ from the latest neutrino oscillation global-fit analysis have been adopted. It can be seen from Fig. 9(a) that maximal $C P$ violation and, as a result, $\mu-\tau$ reflection symmetry, would be ruled out well above $3 \sigma$.

For the other benchmark line of Fig. 9(b) corresponding to maximal $C P$ violation, we find that DUNE has the capability to rule out the possibility of $C P$ conservation at $3 \sigma$. Finally, we also note that the current best-fit point from the global analysis of neutrino oscillation data (marked by the red star) is disfavored at more than $3 \sigma$ for the $C P$ conserving scenario depicted in Fig. 9(a). However, for the maximal $C P$ violating case of Fig. 9(b), the current best-fit point lies within the $2 \sigma$ contour.

Before concluding, let us have a closer look at DUNE's discriminating power on this class of generalized $C P$ theories. For definiteness we discuss in more detail the

\footnotetext{
${ }^{5}$ The benchmark lines are chosen because of their importance and frequent occurrence in the general literature as well as because points on these lines frequently fall within the allowed parameter space of various $C P$ texture zero cases.
} 
cases of benchmark points BP-III [Fig. 7(c)] and BP-VI [Fig. 7(f)]. This will further highlight the discriminating power of DUNE so as to probe, say, the popular $\mu-\tau$ reflection symmetry ansatz. DUNE's potential to rule out certain $C P$ texture zero cases can be best seen by overlaying the plots for the allowed ranges for the $C P$ theories on the DUNE simulated $3 \sigma$ range as shown in Fig. 10. Indeed, one can see that, for any $C P$-motivated true value of Table. II, one can test DUNE's potential to rule out the other $C P$ theory cases which do not include the true value. In the left panel of Fig. 10 (BP-III case) one sees that, after $3.5+3.5 \mathrm{yr}$ of run, DUNE can rule out $X_{I}, X_{V I}, X_{V I I}$, and $X_{X}$ cases at more than $3 \sigma .{ }^{6}$ In the right panel of Fig. 10 (BPVI case), one sees how DUNE can rule out $X_{I}, X_{I V}$, and $X_{I X}$ cases at more than $3 \sigma$, while only a very small part of $X_{V}$ survives at $3 \sigma$. Similar plots can be drawn for all benchmark points listed in Table II, taking the DUNE simulations of Figs. 7 and 8 and comparing them with the allowed ranges for the $C P$ theories in Sec. III.

\section{SUMMARY AND DISCUSSION}

In this paper we have focused on the potential of the DUNE experiment in probing leptonic $C P$ violation. We have adopted the model-independent framework provided by the class of theories with generalized $C P$ symmetries. Using their characterization by the texture zeros of the corresponding generalized $C P$ transformation matrices we have determined the experimental sensitivities for various interesting cases. In order to illustrate our results, we have focused on the two poorly known parameters, i.e., atmospheric mixing angle $\theta_{23}$ and the Dirac $C P$ phase $\delta_{C P}$, for which we have taken various benchmark values as true points in our simulations. The full set of possible benchmark values is given in Table II. We have quantified the capability of the DUNE experiment for various theorymotivated cases assuming $3.5 \mathrm{yr}$ of DUNE running in the

\footnotetext{
${ }^{6}$ Note that case $X_{V I}$ is not shown in Fig. 10 (left panel) in order to avoid clutter.
}

neutrino mode and another $3.5 \mathrm{yr}$ of run in the antineutrino mode. Our results are summarized in Figs. 7-9 for a representative set of benchmark points and lines. We conclude that DUNE will be able to test such theories in a meaningful way, potentially excluding some patterns of texture zeros for the $C P$ transformations.

Before closing we also note that, once DUNE starts taking data, the determination of $\theta_{23}$ and $\delta_{C P}$ will very quickly become DUNE data driven. Thus, if the true value of these parameters is not near the current best-fit values of Ref. [19], then the contours of the allowed regions for a given C.L. will change dramatically as shown in Figs. 7 and 8. In order to highlight the impact of DUNE on the current state of our knowledge of $\theta_{23}-\delta_{C P}$, we have marked the best-fit value of the latest global fit results [19] by a red star. These figures also show how the allowed region will change after $3.5+$ $3.5 \mathrm{yr}$ of DUNE run, if any of the theory predicted benchmark points is indeed the true value of these parameters. For example, for the BP-I point, at the end of the $3.5+3.5 \mathrm{yr}$ run of DUNE, the current best-fit point will be almost $2 \sigma$ away from the true value, as shown in Fig. 7(a), while for BP-II it will be more than $3 \sigma$ away, as evident from Fig. 7(b). Similar conclusions can also be drawn for other benchmark points, as shown in Figs. 7 and 8. In some cases, such as for BP-III and BP-IV, the current best-fit point can be ruled out at more than $\chi^{2}=40$, as shown in Figs. 7(c) and 7(d). Thus, if one of these benchmark points is indeed the true value, DUNE will unambiguously drive the data towards it, away from the current best fit.

\section{ACKNOWLEDGMENTS}

We thank P. Denton for his valuable comments. The research work of N. N. was supported in part by the National Natural Science Foundation of China under Grant No. 11775231. The research work of R. S. and J. W. F. V. was supported by the Spanish Grants FPA2017-85216-P (AEI/FEDER, UE), SEV-2014-0398, PROMETEO/2018/ 165 (Generalitat Valenciana), and the Spanish Red Consolider MultiDark FPA2017-90566-REDC.
[1] T. Kajita, Nobel Lecture: Discovery of atmospheric neutrino oscillations, Rev. Mod. Phys. 88, 030501 (2016).

[2] A. B. McDonald, Nobel Lecture: The Sudbury Neutrino Observatory: Observation of flavor change for solar neutrinos, Rev. Mod. Phys. 88, 030502 (2016).

[3] J. W. F. Valle and J. C. Romao, Neutrinos in High Energy and Astroparticle Physics (John Wiley \& Sons, 2015), http:// eu.wiley.com/WileyCDA/WileyTitle/productCd-3527411976 .html.
[4] V. A. Kuzmin, V. A. Rubakov, and M. E. Shaposhnikov, On the anomalous electroweak baryon number nonconservation in the early Universe, Phys. Lett. 155B, 36 (1985).

[5] M. Dine and A. Kusenko, The Origin of the matterantimatter asymmetry, Rev. Mod. Phys. 76, 1 (2003).

[6] M. Fukugita and T. Yanagida, Baryogenesis without grand unification, Phys. Lett. B 174, 45 (1986).

[7] R. Acciarri et al. (DUNE Collaboration), Long-Baseline Neutrino Facility (LBNF) and Deep Underground Neutrino 
Experiment (DUNE) Conceptual Design Report Volume 1: The LBNF and DUNE Projects, arXiv:1601.05471.

[8] J. Schechter and J.W.F. Valle, Neutrino masses in $\mathrm{SU}(2) \times \mathrm{U}(1)$ theories, Phys. Rev. D 22, 2227 (1980).

[9] S. S. Chatterjee, M. Masud, P. Pasquini, and J. W. F. Valle, Cornering the revamped BMV model with neutrino oscillation data, Phys. Lett. B 774, 179 (2017).

[10] R. Srivastava, C. A. Ternes, M. Tortola, and J. W. F. Valle, Testing a lepton quarticity flavor theory of neutrino oscillations with the DUNE experiment, Phys. Lett. B 778, 459 (2018).

[11] R. Srivastava, C. A. Ternes, M. Tortola, and J. W. F. Valle, Zooming in on neutrino oscillations with DUNE, Phys. Rev. D 97, 095025 (2018).

[12] K. Chakraborty, K. N. Deepthi, S. Goswami, A. S. Joshipura, and N. Nath, Partial $\mu-\tau$ reflection symmetry and its verification at DUNE and hyper-Kamiokande, Phys. Rev. D 98, 075031 (2018).

[13] N. Nath, Consequences of $\mu-\tau$ reflection symmetry at DUNE, Phys. Rev. D 98, 075015 (2018).

[14] T. Fukuyama and H. Nishiura, Mass matrix of Majorana neutrinos, arXiv:hep-ph/9702253.

[15] K.S. Babu, E. Ma, and J. W. F. Valle, Underlying A(4) symmetry for the neutrino mass matrix and the quark mixing matrix, Phys. Lett. B 552, 207 (2003).

[16] P. F. Harrison and W. G. Scott, $\mu-\tau$ reflection symmetry in lepton mixing and neutrino oscillations, Phys. Lett. B 547, 219 (2002).

[17] W. Grimus and L. Lavoura, A nonstandard $C P$ transformation leading to maximal atmospheric neutrino mixing, Phys. Lett. B 579, 113 (2004).

[18] Z.-z. Xing and Z.-h. Zhao, A review of mu-tau flavor symmetry in neutrino physics, Rep. Prog. Phys. 79, 076201 (2016).

[19] P. F. de Salas, D. V. Forero, C. A. Ternes, M. Tórtola, and J. W. F. Valle, Status of neutrino oscillations 2018: $3 \sigma$ hint for normal mass ordering and improved $C P$ sensitivity, Phys. Lett. B 782, 633 (2018).

[20] S. Morisi, D. V. Forero, J. C. Romão, and J. W. F. Valle, Neutrino mixing with revamped $A_{4}$ flavor symmetry, Phys. Rev. D 88, 016003 (2013).

[21] P. Chen, G.-J. Ding, F. Gonzalez-Canales, and J. W. F. Valle, Classifying $C P$ transformations according to their texture zeros: theory and implications, Phys. Rev. D 94, 033002 (2016).

[22] P. Chen, G.-J. Ding, F. Gonzalez-Canales, and J. W. F. Valle, Generalized $\mu-\tau$ reflection symmetry and leptonic $C P$ violation, Phys. Lett. B 753, 644 (2016).
[23] P. Chen, S. C. Chulia, G.-J. Ding, R. Srivastava, and J. W. F. Valle, Realistic tribimaximal neutrino mixing, Phys. Rev. D 98, 055019 (2018).

[24] P. Chen, S. C. Chulia, G.-J. Ding, R. Srivastava, and J. W. F. Valle, Neutrino predictions from generalized $C P$ symmetries of charged leptons, J. High Energy Phys. 07 (2018) 077.

[25] P. Chen, C.-C. Li, and G.-J. Ding, Lepton flavor mixing and CP symmetry, Phys. Rev. D 91, 033003 (2015).

[26] E. Ma and R. Srivastava, Dirac or inverse seesaw neutrino masses with $B-L$ gauge symmetry and $S_{3}$ flavor symmetry, Phys. Lett. B 741, 217 (2015).

[27] E. Ma, N. Pollard, R. Srivastava, and M. Zakeri, Gauge $B-L$ model with residual $Z_{3}$ symmetry, Phys. Lett. B 750, 135 (2015).

[28] S. C. Chulia, E. Ma, R. Srivastava, and J. W. F. Valle, Dirac neutrinos and dark matter stability from lepton quarticity, Phys. Lett. B 767, 209 (2017).

[29] S. C. Chulia, R. Srivastava, and J. W. F. Valle, Seesaw roadmap to neutrino mass and dark matter, Phys. Lett. B 781, 122 (2018).

[30] S. C. Chulia, R. Srivastava, and J. W. F. Valle, Seesaw Dirac neutrino mass through dimension-six operators, Phys. Rev. D 98, 035009 (2018).

[31] J. Schechter and J. W. F. Valle, Neutrino oscillation thought experiment, Phys. Rev. D 23, 1666 (1981).

[32] A. S. Joshipura and K. M. Patel, Pseudo-Dirac neutrinos from flavour dependent $C P$ symmetry, J. High Energy Phys. 07 (2018) 137.

[33] R. Acciarri et al. (DUNE Collaboration), Long-Baseline Neutrino Facility (LBNF) and Deep Underground Neutrino Experiment (DUNE) Conceptual Design Report Volume 2: The Physics Program for DUNE at LBNF, arXiv:1512.06148.

[34] T. Alion et al. (DUNE Collaboration), Experiment Simulation Configurations Used in DUNE CDR, arXiv:1606.09550.

[35] P. Huber, M. Lindner, and W. Winter, Simulation of longbaseline neutrino oscillation experiments with GLoBES (General Long Baseline Experiment Simulator), Comput. Phys. Commun. 167, 195 (2005).

[36] P. Huber, J. Kopp, M. Lindner, M. Rolinec, and W. Winter, New features in the simulation of neutrino oscillation experiments with GLoBES 3.0: General Long Baseline Experiment Simulator, Comput. Phys. Commun. 177, 432 (2007) 432.

[37] P. F. De Salas, S. Gariazzo, O. Mena, C. A. Ternes, and M. Tortola, Neutrino Mass Ordering from Oscillations and Beyond: 2018 Status and Future Prospects, Front. Astron. Space Sci. 5, 36 (2018). 